\title{
PHEMU 2003 campaign: observations of the mutual phenomena of the Galilean satellites in Romania
}

\author{
J.-E. Arlot ${ }^{1}$, G.-D. Chis ${ }^{2, \star}$, L. Farkas ${ }^{3}$, D. Moldovan ${ }^{2}$, A. Nedelcu ${ }^{4}$, P. Popescu ${ }^{4}$, S. Sorescu ${ }^{5}$, M. Stavinschi ${ }^{4}$, \\ L. Serbanescu ${ }^{4}$, V. Tudose ${ }^{4}$, and V. Turcu ${ }^{2}$ \\ ${ }^{1}$ Institut de Mécanique Céleste et Calcul des Éphémerides, 77 avenue Denfert-Rochereau, 75014 Paris, France \\ e-mail: arlot@imcce.fr \\ 2 Astronomical Institute of the Romanian Academy, Str. Ciresilor 19, Cluj-Napoca 3400, Romania \\ e-mail: vturcu@academie.cj .edu.ro \\ 3 Astronomical Institute of the Romanian Academy, Piata Axente Sever 1, Timisoara 1900, Romania \\ 4 Astronomical Institute of the Romanian Academy, Str. Cutitul de Argint 5, Bucharest 040557, Romania \\ e-mail: vtudose@aira.astro.ro \\ 5 “Mircea cel Batran" Naval Academy, Str. Fulgerului 1, Constanta 900218, Romania
}

Received 24 December 2004 / Accepted 1 February 2005

\begin{abstract}
We present CCD, photoelectric, and video observations of selected mutual phenomena of the Galilean satellites. The campaign was carried out in Bucharest and Cluj-Napoca as part of the PHEMU2003 international effort. Five different optical systems were used for data acquisition. Here we report the observational results obtained in acceptable meteorological conditions for twenty mutual phenomena, nine eclipses, and eleven occultations. A preliminary analysis of the observations estimates the accuracy of the data.
\end{abstract}

Key words. planets and satellites: Galilean satellites - astrometry - eclipses - occultations

\section{Introduction}

Twice every 11.8 years Jupiter transits the nodes of its orbit. Because inclination of the orbital planes of Galilean satellites with respect to the planet's equatorial plane is very small, for a few months during these passages, the satellites either occult or eclipse each other, depending on whether they are collinear with the Earth or with the Sun, respectively. These events are referred to as mutual phenomena (see Arlot 2002b). Their observation can be used, for instance, to derive corrections of the orbital parameters or to study the surface properties of involved jovian satellites.

The first observational campaign of the mutual phenomena took place in 1973 and was followed later by five other international collaborations in 1979, 1985, 1991, 1997, and 2003. Thuillot \& Tanga (1997) give a thorough presentation of the published papers that made use of the data gathered on all these occasions (except for 2003). The Astronomical Institute of the Romanian Academy participated actively in these three projects: PHEMU1991 (Oprescu et al. 1992), PHEMU1997 (Vass 1999; Vass \& Serbanescu 1999; Dumitrescu et al. 1999), and PHEMU2003 (this work).

\section{Observations}

We give below characteristics of the material used in the different observation sites.

a. Bucharest Observatory (Long.: $1^{\mathrm{h}} 44^{\mathrm{m}} 23.115$ E; Lat: $44^{\circ} 24^{\prime} 50^{\prime} .4 \mathrm{~N}$; Alt.: $80 \mathrm{~m}$ ).

For data acquisition four different optical systems were used:

SYS1: SBIG ST6-V CCD camera $(375 \times 242$ pixels, $23 \times 27 \mu \mathrm{m}$ pixel size) with Johnson $V$ filter mounted on a $15 \mathrm{~cm}$ diameter, $f / d=19$ Cassegrain telescope.

SYS2: HiSis22 CCD camera on the visual refractor of the Prin-Mertz double astrograph with a $38 \mathrm{~cm}$ diameter, $f / d=16$.

SYS3: photoelectric photometer with EMI 9502B photomultiplier, in the primary focus of a $50 \mathrm{~cm}$ diameter, $f / d=$ 15 Cassegrain telescope.

SYS4: Mintron CCD TV $12 V$ camera $(740 \times 555$ pixels, $9 \times$ $9 \mu \mathrm{m}$ pixel size) mounted on a $25 \mathrm{~cm}$ diameter, $f / d=$ 10 Meade LX50 Schmidt-Cassegrain telescope.

In each case, the data were reduced using a different software package: Astrol (SYS1), Iris (SYS2), or Muffy (SYS4, Nedelcu et al. 2003). 
Table 1. Results of the PHEMU2003 campaign in Romania. In column 2, E and O denote eclipses and occultations, respectively. Times in Cols. 3 and 5 indicate the maxima of each event. For the last two columns see discussion in the text.

\begin{tabular}{|c|c|c|c|c|c|c|c|}
\hline Fig. No & $\begin{array}{l}\text { Date } \\
(\mathrm{mm} / \mathrm{dd} / \text { yyyy })\end{array}$ & Phenomenon & $\begin{array}{l}\text { Predicted time } \\
\text { (TT) }\end{array}$ & $\begin{array}{l}\text { Optical } \\
\text { setup }\end{array}$ & $\begin{array}{l}\mathrm{O}-\mathrm{C}(\mathrm{KW}) \\
(\mathrm{s})\end{array}$ & $\begin{array}{l}\mathrm{O}-\mathrm{C}(\mathrm{P}) \\
(\mathrm{s})\end{array}$ & Observers $^{a}$ \\
\hline 1 & $11 / 12 / 2002$ & $\mathrm{~J} 4 \mathrm{O} \mathrm{J} 2$ & $23 \mathrm{~h} 09 \mathrm{~m} 30 \mathrm{~s}$ & SYS5 & $-16 \pm 0.7$ & $-13 \pm 5.8$ & VLT, DM, GC \\
\hline 2 & $11 / 28 / 2002$ & $\mathrm{~J} 2 \mathrm{E} \mathrm{J} 1$ & $21 \mathrm{~h} 36 \mathrm{~m} 22 \mathrm{~s}$ & SYS5 & $+2 \pm 0.5$ & $+8 \pm 4.4$ & VLT, DM, GC \\
\hline 3 & $11 / 28 / 2002$ & $\mathrm{~J} 2 \mathrm{O} \mathrm{J} 1$ & $23 \mathrm{~h} 41 \mathrm{~m} 24 \mathrm{~s}$ & SYS5 & $-14 \pm 1.2$ & $-9 \pm 12.3$ & VLT, DM, GC \\
\hline 4 & $12 / 13 / 2002$ & $\mathrm{~J} 2 \mathrm{E} \mathrm{J} 1$ & $02 \mathrm{~h} 24 \mathrm{~m} 01 \mathrm{~s}$ & SYS1 & $+1 \pm 0.3$ & $+1 \pm 2.7$ & VAT, SS \\
\hline 5 & $12 / 16 / 2002$ & $\mathrm{~J} 4 \mathrm{O} \mathrm{J} 1$ & $02 \mathrm{~h} 11 \mathrm{~m} 35 \mathrm{~s}$ & SYS5 & $-21 \pm 0.2$ & $-20 \pm 2.6$ & VLT, DM, GC \\
\hline 6 & $12 / 20 / 2002$ & J2 E J1 & $04 \mathrm{~h} 52 \mathrm{~m} 31 \mathrm{~s}$ & SYS5 & $0 \pm 0.2$ & $0 \pm 0.9$ & VLT, DM, GC \\
\hline 7 & $01 / 19 / 2003$ & $\mathrm{~J} 4 \mathrm{O} \mathrm{J} 3$ & $01 \mathrm{~h} 06 \mathrm{~m} 55 \mathrm{~s}$ & SYS5 & $-12 \pm 3.0$ & & VLT, DM, GC \\
\hline 8 & $02 / 03 / 2003$ & J4 O J1 & $17 \mathrm{~h} 13 \mathrm{~m} 12 \mathrm{~s}$ & SYS5 & $-4 \pm 0.9$ & $-7 \pm 2.1$ & VLT, DM, GC \\
\hline 9 & 02/03/2003 & $\mathrm{J} 2 \mathrm{O} \mathrm{J} 3$ & $23 \mathrm{~h} 31 \mathrm{~m} 56 \mathrm{~s}$ & SYS5 & $+15 \pm 1.4$ & $+11 \pm 10.6$ & VLT, DM, GC \\
\hline 9 & $02 / 03 / 2003$ & J2 E J3 & $23 \mathrm{~h} 40 \mathrm{~m} 40 \mathrm{~s}$ & SYS5 & $-3 \pm 1.0$ & $-10 \pm 10.1$ & VLT, DM, GC \\
\hline 10 & $02 / 18 / 2003$ & $\mathrm{~J} 4 \mathrm{O} \mathrm{J} 3$ & $17 \mathrm{~h} 54 \mathrm{~m} 44 \mathrm{~s}$ & SYS5 & $+5 \pm 0.8$ & $+4 \pm 9.4$ & VLT, DM, GC \\
\hline 11 & $02 / 18 / 2003$ & J4 E J3 & $20 \mathrm{~h} 49 \mathrm{~m} 53 \mathrm{~s}$ & SYS5 & $-22 \pm 0.6$ & $-23 \pm 13.2$ & VLT, DM, GC \\
\hline 12 & $02 / 27 / 2003$ & $\mathrm{~J} 1 \mathrm{O} \mathrm{J} 2$ & $17 \mathrm{~h} 49 \mathrm{~m} 00 \mathrm{~s}$ & SYS5 & $-14 \pm 0.4$ & $-15 \pm 1.2$ & VLT, DM, GC \\
\hline 13 & $02 / 27 / 2003$ & $\mathrm{~J} 1 \mathrm{O}$ J4 & $22 \mathrm{~h} 04 \mathrm{~m} 00 \mathrm{~s}$ & SYS5 & $-21 \pm 1.0$ & $-28 \pm 3.4$ & VLT, DM, GC \\
\hline 14 & $02 / 28 / 2003$ & J1 E J4 & $01 \mathrm{~h} 17 \mathrm{~m} 12 \mathrm{~s}$ & SYS5 & $-33 \pm 0.8$ & $-33 \pm 5.6$ & VLT, DM, GC \\
\hline 15 & $02 / 28 / 2003$ & $\mathrm{~J} 1 \mathrm{O}$ J4 & $23 \mathrm{~h} 46 \mathrm{~m} 13 \mathrm{~s}$ & SYS5 & $-20 \pm 0.6$ & $-17 \pm 33.8$ & VLT, DM, GC \\
\hline 16 & $03 / 24 / 2003$ & J1 E J3 & $22 \mathrm{~h} 02 \mathrm{~m} 36 \mathrm{~s}$ & SYS3 & $-31 \pm 1.4$ & $-24 \pm 8.2$ & VAT, SS \\
\hline 17 & 04/02/2003 & J2 E J1 & $22 \mathrm{~h} 59 \mathrm{~m} 05 \mathrm{~s}$ & SYS4 & $-11 \pm 1.3$ & & $\mathrm{AN}$ \\
\hline 18 & $04 / 03 / 2003$ & J1 E J4 & $17 \mathrm{~h} 23 \mathrm{~m} 58 \mathrm{~s}$ & SYS4 & $-40 \pm 1.5$ & & $\mathrm{AN}$ \\
\hline 19 & $04 / 21 / 2003$ & $\mathrm{~J} 1 \mathrm{O} \mathrm{J} 2$ & $21 \mathrm{~h} 30 \mathrm{~m} 27 \mathrm{~s}$ & SYS2 & $-54 \pm 1.5$ & & LS \\
\hline
\end{tabular}

${ }^{a}$ GC: G.-D. Chis; DM: D. Moldovan; AN: A. Nedelcu; SS: S. Sorescu; LS: L. Serbanescu; VAT: V. Tudose; VLT: V. Turcu.

b. Cluj-Napoca Observatory (Long.: $1^{\mathrm{h}} 34^{\mathrm{m}} 22^{\mathrm{s}} .47$ E; Lat.: $46^{\circ} 42^{\prime} 36^{\prime \prime} 3 \mathrm{~N}$; Alt.: $\left.756 \mathrm{~m}\right)$

SYS 5 : Meade Pictor 416 CCD camera $(384 \times 256$ pixels, $18 \times 18 \mu \mathrm{m}$ pixel size) with $V$ filter (G-550, Meade Pictor 616 filter wheel) on a $40 \mathrm{~cm}$ diameter, $f / d=10$ Meade LX200 Schmidt-Cassegrain telescope.

The images were reduced with AIP4WIN.

c. Timisoara Observatory (Long.: $1^{\mathrm{h}} 24^{\mathrm{m}} 55^{\mathrm{s}} 33$ E; Lat.: $45^{\circ} 44^{\prime} 17^{\prime \prime} .58 \mathrm{~N}$; Alt.: $90 \mathrm{~m}$ )

For this campaign, the $30 \mathrm{~cm} \mathrm{f} / \mathrm{d}=18$ Cassegrain telescope was endowed with a ST-237 CCD camera $(640 \times 480$ pixels, $7.4 \times 7.4 \mu \mathrm{m}$ pixel size) by IMCCE. Due to the overly long bureaucracy, this telescope was upgraded too late to participate in the campaign.

The error in synchronization with a time base was of $0.5 \mathrm{~s}$ for SYS2, SYS4, and SYS5, 2 s for SYS1, and $5 \mathrm{~s}$ for SYS3. The light curves observed during the campaign are presented in Figs. 1-19.

\section{Results}

The observed times of minimum light (i.e. the maximum of the events) were determined using two different methods.

First, with the Kwee-van Woerden (KW) method (Kwee \& van Woerden 1956) we implicitly considered no asymmetry between the ascending and descending wings of the light curves for an attempt to approximate the profiles of the light curves with analytical functions, see e.g. Froeschle et al. (1992). The fitted values, in the form of $(\mathrm{O}-\mathrm{C})$ 's calculated from the predicted time, and the corresponding errors are shown in Col. 5 of Table 1. The predicted geometric mid-times are from Arlot (2002a).

Second, the results of polynomial fitting $(\mathrm{P})$ of the lightcurve. The results, in the form of $(\mathrm{O}-\mathrm{C})$ 's referred to the predicted time, are provided in Col. 6 of Table 1. Errors are larger than with the previous method since they are very sensitive to dispersion of the points on the light curves. If the points are integrated as in the the light curve of Figs. 5, 6, the error is around one to three seconds in time. If the points are more numerous and not integrated such as in Fig. 15, the error is around $30 \mathrm{~s}$ in time.

The residuals (O-Cs) listed in Table 1 are for illustrative purposes only and have no physical meaning in the strict sense. Due to the solar phase angle (the angle Sun-satellites-Earth), there is a time lag between the light minimum (determined from the light curves) and the geometric minimum, i.e. the time of closest approach of the geometric centers of the satellites (e.g. Vasundhara 1991). Moreover, the uneven surface brightness of the disks has to be accounted for (Aksnes et al. 1986; Vasundhara 1994), along with the different time systems used in Cols. 3 and 5 of Table 1. For all these reasons stated above, the residuals we present should be treated with care and considered as a first estimation of the accuracy of the observations by comparing the two different methods of reduction. Note that the velocity of the satellites does not exceed $10 \mathrm{~km} \mathrm{~s}^{-1}$ and allows conversion of the $(\mathrm{O}-\mathrm{C})$ 's in kilometers. Then the $(\mathrm{O}-\mathrm{C}) \mathrm{s}$ range from 0 to $330 \mathrm{~km} \mathrm{~s}$, with a few larger values of 


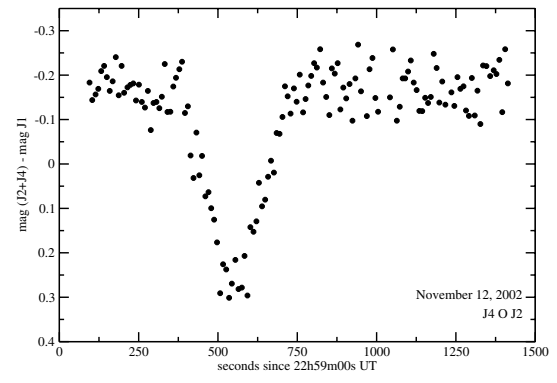

Fig. 1. J4 occults J2 on November 12, 2002.

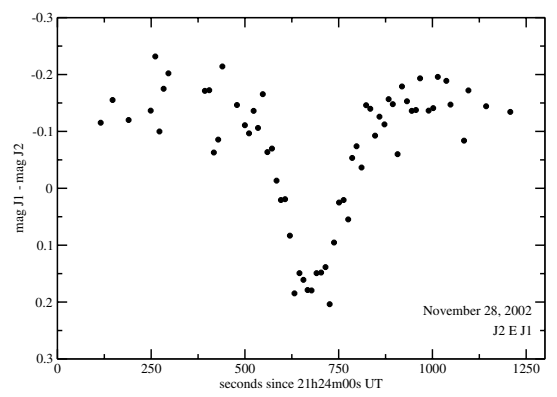

Fig. 2. J2 eclipses J1 on November 28, 2002.

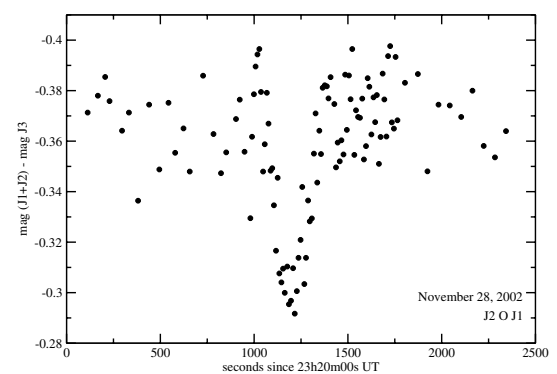

Fig. 3. J2 occults J1 on November 28, 2002.

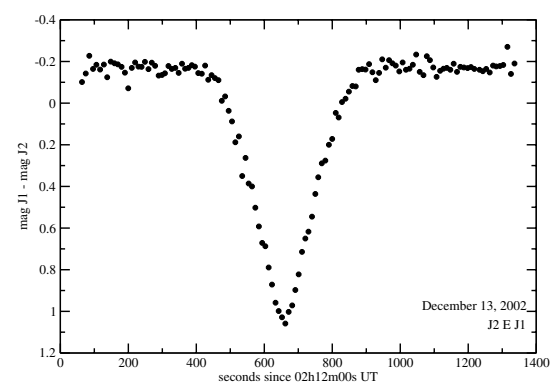

Fig. 4. J2 eclipses J1 on December 13, 2002.

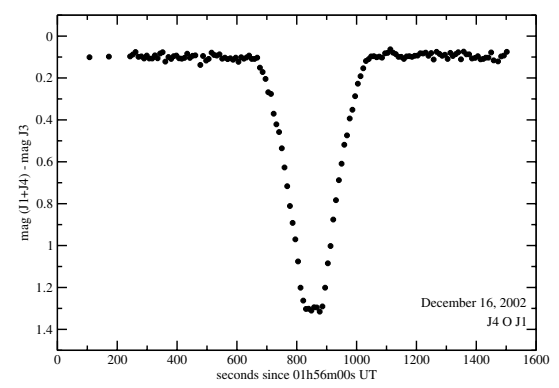

Fig. 5. J4 occults J1 on December 16, 2002.

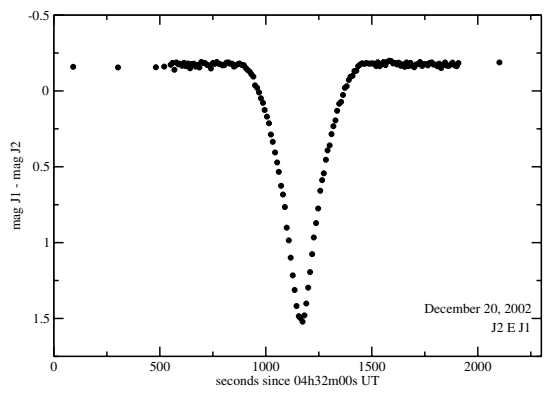

Fig. 6. J2 eclipses J1 on December 20, 2002.

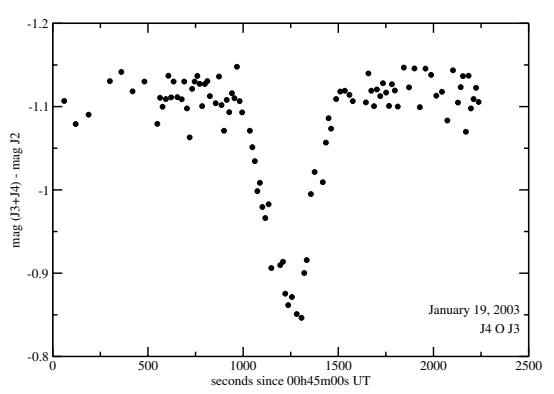

Fig. 7. J4 occults J3 on January 19, 2003.

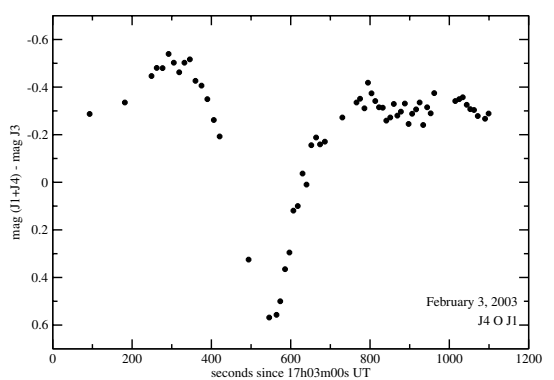

Fig. 8. J4 occults J1 on February 3, 2003.

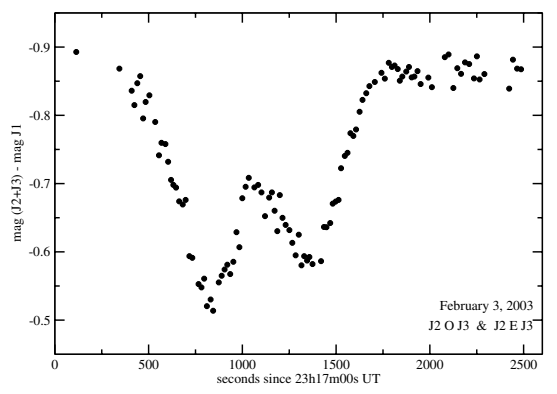

Fig. 9. J2 occults and eclipses J3 on February 3, 2003.

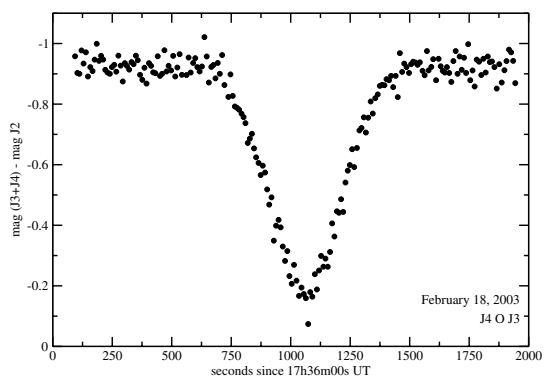

Fig. 10. J4 occults J3 on February 18, 2003. 


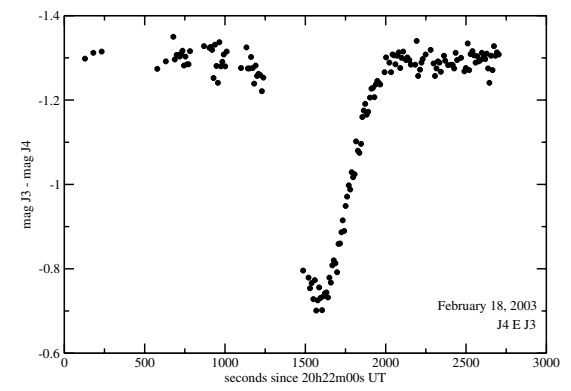

Fig. 11. J4 eclipses J3 on February 18, 2003.

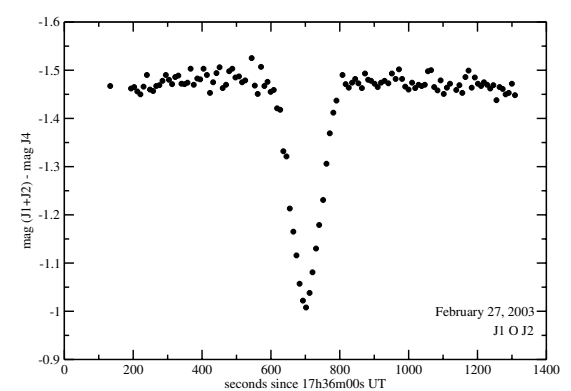

Fig. 12. J1 occults J2 on February 27, 2003.

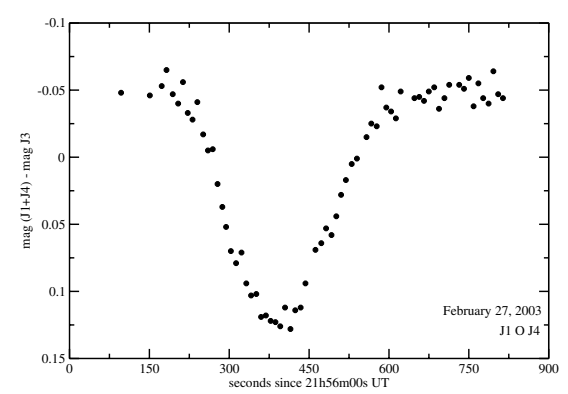

Fig. 13. J1 occults J4 on February 27, 2003.

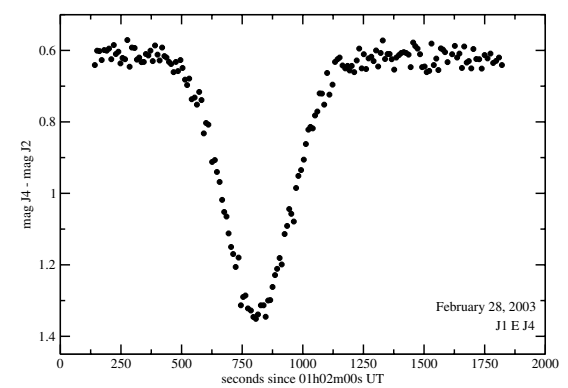

Fig. 14. J1 eclipses J4 on February 28, 2003.

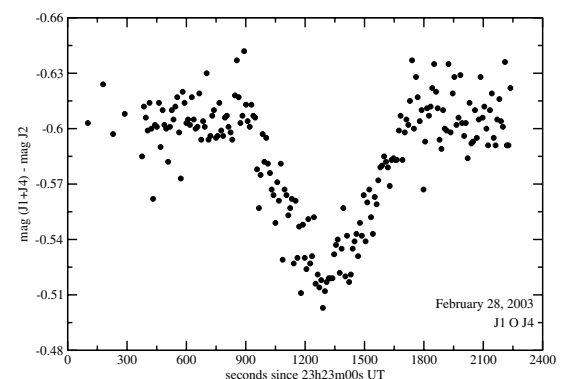

Fig. 15. J1 occults J4 on February 28, 2003.

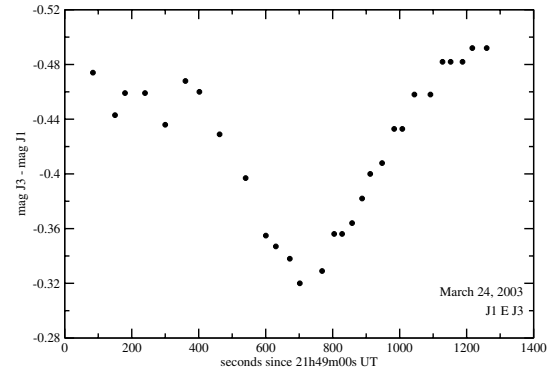

Fig. 16. J1 eclipses J3 on March 24, 2003.

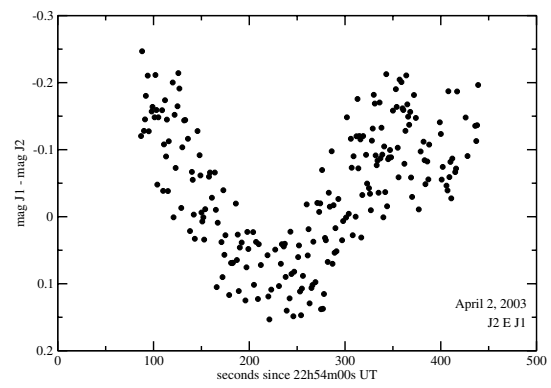

Fig. 17. J2 eclipses J1 on April 2, 2003.

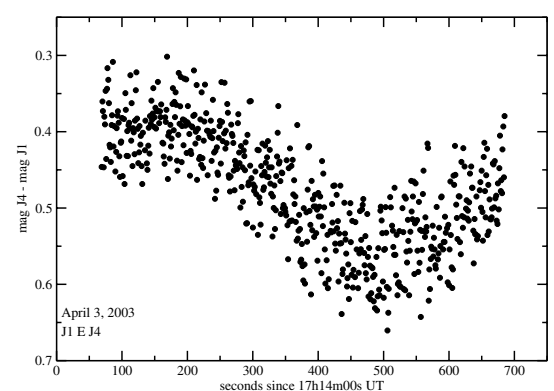

Fig. 18. J1 eclipses J4 on April 3, 2003.

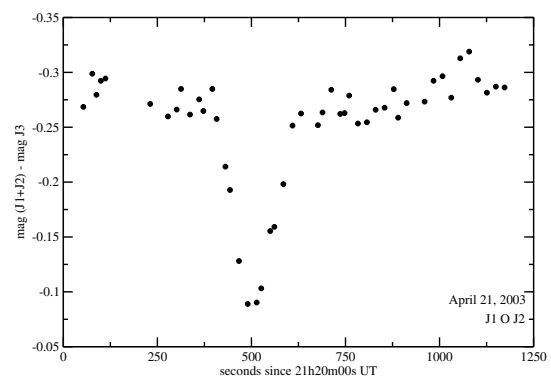

Fig. 19. J1 occults J2 on April 21, 2003.

400 and $540 \mathrm{~km} \mathrm{~s}$. Converted into arcseconds, it ranges from 0 to 0.1 arcsec. In fact, the accuracy is the same as that in direct astrometric observations, but can be improved if taking the surface brightness of the satellites into account, along with the phase defect.

\section{Conclusions}

Above we only use the time shift without taking the drop in magnitude into account. In a few cases (Figs. 3, 8, 11, 12, $14,18)$, values of the magnitude difference on the plateau (i.e. 
before and after the event) were more than 0.1 mag different with respect to the calculated ones. Also, for some phenomena (Figs. 1, 5, 6, 8-10) the magnitude drop during the mutual event was notably inconsistent with predicted values. Since there is no evident correlation between the two tendencies, we cannot exclusively consider the quality of data or instrumental effects as the solely cause of the deviations from theory. Further analysis of this topic is underway.

\section{References}

Aksnes, K., Franklin, F., \& Magnusson, P. 1986, AJ, 92, 1436

Arlot, J.-E. 2002a, A\&A, 383, 719

Arlot, J.-E. 2002b, PHEMU Technical Note No. 1, Institute for celestial mechanics and ephemerid calculation, Paris
Dumitrescu, A., Stefanescu, G., Sorescu, S. 1999, Rom. Astron. J., 9, 79

Froeschle, M., Meyer, C., \& Mignard, F. 1992, A\&A, 262, 308

Kwee, K. K., \& van Woerden, M. 1956, Bull. Astron. Inst. Nether., 12, No. 464, 327

Nedelcu, A., Popescu, R., \& Badescu, O. 2003, Proc. South-Eastern Europe Youth at the Telescope Summer School, Belogradchik, Bulgaria, in press

Oprescu, G., Dumitrescu A., Suran, M. D., et al. 1992, Rom. Astron. J., 2, 61

Thuillot, W., \& Tanga, P. 1997, PHEMU Technical Note No. 2, Institute for celestial mechanics and ephemeride calculation, Paris Vass, G. 1999, Rom. Astron. J., 9, 49

Vass, G., \& Serbanescu, L. 1999, Rom. Astron. J., 9, 71

Vasundhara, R. 1991, A\&A 12, 69

Vasundhara, R. 1994, A\&A, 281, 565 